\title{
Tendencias de la innovación mediática en Estados Unidos
}

\author{
MHCJ no 6 | Año 2015 \\ Artículo no 7 (68) \\ Páginas 161 a 193 \\ mhjournal.org
}

\author{
José María Valero Pastor | jose.valeropa@gmail.com \\ Universidad Miguel Hernández de Elche
}

\section{Palabras clave}

innovación mediática, Estados Unidos, mercados comunicativos, tecnologías de la información, organización empresarial

\section{Sumario}

1. Introducción

2. Marco teórico 2.1. Concepto de innovación 2.2. Tipologías de la innovación mediática

3. Metodología 3.1. Panel de expertos 3.2. Criba y selección de casos 3.3. Análisis cualitativo de las iniciativas

4. Resultados 4.1. Base de la innovación 4.2. Objeto de la innovación 4.2.1.

Innovaciones en el producto 4.2.2.

Innovaciones en los procesos de producción y distribución 4.2.3. Innovaciones en la organización empresarial 4.2.4.

Innovaciones en la comercialización

5. Conclusiones

6. Bibliografía

\section{Resumen}

El mercado de la comunicación está siendo objeto de continuas transformaciones. La implantación de nuevas tecnologías y dispositivos, los cambios en los hábitos de consumo de la audiencia, y la actual situación económica obligan a los medios de comunicación a innovar para continuar desempeñando un papel relevante en la sociedad. En el mercado mediático estadounidense, existen entidades como la Knight Foundation, que promueven la innovación, y que, junto a la voluntad renovadora de muchas empresas, provocan que los medios norteamericanos sean vistos como una referencia a nivel mundial. Este artículo analiza cuáles son las principales tendencias de la innovación seguidas por los medios de comunicación de Estados Unidos. Para ello, se ha seleccionado una muestra de casos relevantes. Posteriormente, se emplea una metodología propuesta por Carvajal et al., que clasifica la innovación periodística en cuatro áreas: producto, procesos, organización empresarial y comercialización. Este método fue creado para valorar las innovaciones también según su grado de ruptura con los patrones del mercado. Sin embargo, el presente trabajo no tiene en cuenta este factor, por lo que la metodología ha sido adaptada a los objetivos de la investigación: identificar tendencias generales de innovación periodística. Además, la investigación ofrece una mirada crítica hacia el mercado mediático español, pues otro de los objetivos es comparar la innovación implementada en él con aquellas iniciativas adoptadas por los medios de comunicación estadounidenses. Cabe destacar a este respecto la utilización del informe Ranking de Innovación Periodística 2014, publicado por el Grupo de Investigación en Comunicación de la Comunidad Valenciana, de la Universidad Miguel Hernández de Elche.

\section{Forma de citar este artículo en las bibliografías}

José María Valero Pastor (2015): “Tendencias de Innovación Mediática en los Estados Unidos”, en Miguel Hernández Communication Journal, nº, páginas 161 a 193. Universidad Miguel Hernández, UMH (Elche-Alicante). Recuperado el _ de de 20__ de: @ink del artículo en mhjournal.org] 


\author{
José María Valero Pastor | jose.valeropa@gmail.com \\ Universidad Miguel Hernández de Elche
}

\author{
MHCJ nㅇ 6 | Year 2015 \\ Paper no 7 (68) \\ Pages 161 to 193 \\ mhjournal.org
}

Keywords

media innovation, United States, media markets, information technologies, business organization

Sumary

1. Introduction.

2. Theoretical Framework. 2.1. Innovation Concept 2.2. Media Innovation Typology

3. Methodology 3.1. Expert Panel 3.2. Filter and Selection of Cases 3.3. Qualitative Analysis of the Initiatives

4. Results 4.1. Innovation Base 4.2. Innovation Object 4.2.1. Product Innovation 4.2.2. Production and Distribution Processes Innovation 4.2.3. Business Innovation 4.2.4. Marketing Innovation

5. Conclusions

6. Bibliography

\section{Abstract}

In recent years, media market undergoes continuous transformations. The implementation of new technologies and devices, the changes in consumption patterns of the audience and the current economic situation force media to innovate in order to continue to perform a significant role in society. In the United States media market, there are organizations such as the Knight Foundation, that promote innovation, and that, along with the renewal willingness of many companies, cause North American media are seen as a global benchmark in this area.

This final degree work analyzes the main trends of innovation adopted by the media in the United States. The first step in order to do this is to select a sample of startups and media to undergo the analysis. A methodology proposed by Carvajal et al. is employed afterwards. This methodology classifies the journalistic innovation into four broad areas: product, production and distribution processes, business organization and marketing. This method was created with the additional aim of evaluating the innovations according to their degree of rupture with market patterns. However, this study does not take into account this factor, so the methodology has been adapted to the research objectives: identify general trends in media innovation.

In addition, this research offers a critical look at the Spanish media market, as one of the stated objectives is to compare the innovative initiatives implemented in it with those innovations adopted on a regular basis by the United State media. It is noteworthy the use of Ranking de Innovación Periodística 2014 report, published by the Grupo de

Investigación en Comunicación de la Comunidad Valenciana of Elche’s Miguel Hernández University. This document is used as the main reference for the conclusions of the work in order to establish the similarities and differences between both media markets.

\title{
How to cite this paper in bibliographies
}

José María Valero Pastor (2015): "Media innovation trends in the United States”, en Miguel Hernández

Communication Journal, nº, pages 161 to 193. Universidad Miguel Hernández, UMH (Elche-Alicante). Recuperado el__de de 20_ de: [ink del artículo en mhjournal.org] 


\section{Introducción}

La presente investigación tiene como objeto principal de estudio la innovación en los medios de comunicación estadounidenses. La innovación se entiende en la actualidad como la mayor baza en la lucha por conseguir la supervivencia de la industria mediática en un contexto económico y social nada favorable para la continuidad de las viejas prácticas periodísticas.

En este contexto, la rápida implantación de Internet juega un papel importante, pues es señalado como uno de los causantes de la crisis de los modelos periodísticos tradicionales. Según el Libro blanco de la prensa diaria 2014, publicado por la Asociación de Editores de Diarios Españoles (AEDE), el número de españoles que consumen medios de comunicación exclusivamente en internet ha aumentado de 144.000 en 2001 a cuatro millones en 2014. Este dato contrasta con las cifras de difusión de diarios impresos, que muestran un decremento del $29,6 \%$ desde el año 2001.

En Estados Unidos, la venta de prensa escrita también retrocede, incluso entre los medios con más relevancia. El diario de referencia, The New York Times, disminuyó su difusión de los 1,1 millones de ejemplares en las ediciones diarias en 2002 a los 928.000 en 2009. Contrasta con estas cifras el dato aportado por Arthur O. Sulzberger, editor del diario, que en mayo de 2014 aseguraba en una charla en IESE Business School que la edición digital de The New York Times había alcanzado los 65 millones de visitantes únicos al mes. The Washington Post también registró un descenso en la difusión, que en 2002 era de 768.000 ejemplares, mientras que en 2009 la cifra cayó a 582.000 (Martínez Molina, 2010).

Esta tendencia al alza de Internet en detrimento de los medios en formato papel se percibe también en términos de inversión publicitaria. Según demuestra el informe State of the News Media, elaborado por Pew Research Center, los ingresos por patrocinio en prensa escrita han descendido más de un 4\% hasta situarse en 16.400 millones de dólares en 2014, mientras que en 2005 se alcanzaba una cifra de 47.400 millones. Al mismo tiempo, la publicidad digital aumenta un 18\%, con lo que los ingresos se sitúan en 50.700 millones de dólares. Dentro de esta cifra, tiene un peso relevante la publicidad en dispositivos móviles, que supone un 37\% de toda la publicidad digital, doce puntos porcentuales más que en el año 2013.

La aparición de nuevos dispositivos como smartphones y tablets modifican los hábitos de consumo del periodismo (Carvajal, 2012). La penetración de estos aparatos es cada vez mayor. La Encuesta sobre equipamiento y uso de tecnologias de comunicación e información en los hogares $2014 \mathrm{del}$ Instituto Nacional de Estadística muestra que, por primera vez, "el principal tipo de conexión a Internet por banda ancha es la conexión móvil a través de un dispositivo de mano (...) con un $67,2 \%$ de los hogares con acceso".

En Estados Unidos, según una encuesta sobre el uso de los smartphones en 2015 realizada por Pew Research Center para su informe State of News Media, un 46\% de la población que está en posesión de un teléfono inteligente afirma que "no podría vivir sin él". Además, la misma 
investigación revela que el móvil es ya el primer soporte de acceso a medios de comunicación en Internet, pues 39 de cada 50 sitios de noticias reciben más tráfico desde estos dispositivos que desde ordenadores.

Estas circunstancias, además de otros factores económicos, sociológicos o de hábitos de consumo, dan lugar a un nuevo panorama mediático. Los medios de comunicación deben superar la crisis en la que se han visto inmersos durante los últimos años. Por ello se hace necesario innovar, entendiendo la innovación como una creación de valor añadido para que el producto periodístico aparezca atractivo a los ojos del consumidor, pero también como la búsqueda de un modelo que permita la rentabilidad económica y el ahorro de costes de producción y distribución. Por este motivo, se hace necesario innovar y, por consiguiente, estudiar y comprender el concepto de innovación para poder implantarlo.

Sin embargo, y aunque a veces la relación sea estrecha, no se debe confundir innovación con emprendimiento. Empresas mediáticas altamente arraigadas en el mercado como The New York Times o The Washington Post han entendido la necesidad de innovar para que su producto perdure. A este respecto, uno de los documentos más notorios en el ámbito de la innovación mediática en 2014 fue el Media Innovation Report. Se trata de un informe filtrado a la prensa especializada en el que un equipo de The New York Times analiza las claves del cambio del mercado mediático estadounidense, y ofrece unas pautas sobre cómo la dama gris debe adaptarse a él.

Los medios estadounidenses fueron los primeros en percibir algunas de las necesidades del nuevo mercado de la comunicación. Cabeceras tradicionales hiperlocales como el News \& Observer habían conseguido ya en 2008 una convergencia total entre las redacciones impresa y digital, así como la creación de una red de blogs asociados a su marca (González Esteban, 2009), algo que sus homólogos españoles todavía no han conseguido plenamente en la actualidad. Por lo tanto, cabe pensar que las claves de la innovación periodística que se acaban adoptando en España vienen definidas por la actuación de los medios norteamericanos; o al menos, estas podrían servir de pauta de actuación para los próximos años.

Por último, estos esfuerzos por entender el concepto y las tendencias de la innovación han tenido un hito relevante que sirve de marco y guía para el presente trabajo. El 20 de enero de 2015, el Grupo de Investigación en Comunicación de la Comunidad Valenciana (GICOV) publicó su Ranking de innovación periodística 2014. En él se identifican las principales iniciativas innovadoras en los medios de comunicación españoles, mediante lo cual se ofrece al lector un boceto del ecosistema mediático en España. El informe emplea una metodología que sistematiza el análisis de la innovación periodística de una forma holista, que pretende abarcar todas las áreas en las que es posible innovar en los medios.

El propósito de este trabajo es definir con claridad las tendencias innovadoras perfiladas por los medios de comunicación estadounidenses en los últimos años. 
Para cumplir los objetivos anteriormente citados, se analizarán de forma pormenorizada las iniciativas innovadoras adoptadas por los medios de comunicación de Estados Unidos. Se adoptará para ello una metodología de análisis conveniente, que se adapte a las necesidades y las peculiaridades del tema tratado y de las metas estipuladas.

\section{Marco teórico}

Como se explica en la introducción, el ecosistema mediático se encuentra sumido en un contexto metamórfico en los últimos años. Por ello, la atención de la comunidad académica periodística se dirige, cada vez más, hacia el fenómeno de la innovación en medios de comunicación (Bleyen et al., 2014). Los esfuerzos de los investigadores se centran en definir qué es innovación en el sentido estricto del término, además de conseguir un modelo de análisis que ayude a sistematizar su estudio. En este epígrafe, el presente trabajo revela qué entienden los académicos por innovación, además de qué métodos proponen para su análisis.

\subsection{Concepto de innovación}

A continuación, el presente trabajo se centra en definir qué es la innovación, y qué tiene de especial este concepto aplicado a la industria de los medios de comunicación. Para ello, se revisan las definiciones correspondientes a innovación y, en concreto, a innovación mediática, aportada por los académicos y profesionales del sector.

Joseph Schumpeter fue uno de los primeros economistas en abordar el concepto de innovación, en 1934. Para él, la innovación significa una nueva forma de combinar factores productivos ya existentes. Sin embargo, autores más actuales como Dogruel critican esta definición, pues de ser cierta, "cada nuevo título de película respondería a los atributos de una innovación, ya que se agrupa una configuración única de factores de producción (ej. actores, contenido, organización)" (2014: 4). Poco después, el profesor Peter Drucker. Este autor definía la innovación como "la búsqueda, organizada y con un objetivo, de cambios, y el análisis sistemático de las oportunidades que ellos pueden ofrecer para la innovación social o económica" (1997: 64).

Por su parte, la Guía para la recogida e interpretación de datos sobre innovación, elaborada en 2006 por la OCDE y el Eurostat, innovación es "la introducción de un nuevo, o significativamente mejorado, producto (bien o servicio), de un proceso, de un nuevo método de comercialización o de un nuevo método organizativo, en las prácticas internas de la empresa, la organización del lugar de trabajo o las relaciones exteriores". Esta definición de inspiración schumpeteriana revela, además, aquellas facetas en las que se puede innovar en cualquier materia comercial, según el organismo internacional. 
Storsul y Krumsvik (2013) aportan otro matiz al concepto. Para ellos, la innovación consiste en "introducir algo nuevo al sistema socioeconómico" (p. 14), y añaden que no es necesario que ese ítem novedoso sea un "invento", sino que basta con que se trate de una nueva combinación de ideas existentes, siempre y cuando se abran nuevas posibilidades en el mercado. Se acota con esta puntualización la amplia definición de Schumpeter, por lo que se consiguen superar las críticas.

José Alberto García Avilés (2014), profesor titular de periodismo en la Universidad Miguel Hernández, explica en un artículo publicado en el blog del Máster en Innovación en Periodismo que "innovar consiste en detectar un problema o necesidad, encontrar una solución original y desarrollarla de forma exitosa". Este autor establece siete áreas en las que se puede innovar: contenido, audiencia, servicio, producto, tecnología, marketing y modelo de negocio. Para que se pueda considerar que existe innovación en un proyecto, es necesario que este presente cambios en, al menos, dos de los ámbitos de los mencionados. Para exponer su tesis, García Avilés cita varios ejemplos. Uno de ellos es el medio español Acuerdo, que introduce cambios en cuanto a sus contenidos y su modelo de negocio, por lo cual puede ser considerado un medio innovador.

En cuanto al estudio de la innovación, Fagerberg (2005) afirma que, a pesar de no ser un fenómeno nuevo, el concepto no ha recibido toda la atención que se merece por parte de la comunidad académica. No obstante, el autor explica que el interés es creciente y que, por ello, en la primera década del milenio se ha observado una tendencia al alza en el número de publicaciones que tienen a la innovación como objeto. Además, se percibe un viraje del punto de vista de las investigaciones académicas hacia el cruce de disciplinas. Ya no solo se estudia la innovación como un fin en sí mismo, o en relación con la tecnología, sino que se aplica el concepto a otras ciencias sociales y humanas, como la comunicación.

Leyla Dogruel (2014) reivindica la necesidad de establecer un campo de investigación diferenciado para la innovación en comunicación, ya que esta presenta unos rasgos como objeto de estudio que la alejan de otros tipos de innovación. Estas características son cuatro: en primer lugar, en el mercado mediático existe una necesidad constante de innovación porque los productos suelen tener ciclos de vida cortos; en segundo lugar, la innovación mediática presenta un alto riesgo, pues los costes de adopción de iniciativas suelen ser altos y los ingresos dependen de una audiencia volátil; en tercer lugar, la innovación mediática es fruto de una estrecha interacción entre las ideas creativas y los aspectos tecnológicos y organizacionales; y, por último, la frontera entre innovación en el producto e innovación en el proceso pueden ser difusas e inidentificables.

Los esfuerzos de los académicos por establecer un estudio de la innovación en el ámbito del periodismo consiguen sus frutos con la aparición de investigaciones, tanto académicas como de la industria periodística, para definir qué iniciativas son más convenientes para su adopción en el mercado de la comunicación. En cuanto a los análisis realizados por grupos de 
investigadores, destacan el State of the News Media, realizado por el Pew Research Center desde su fundación en 2004, o el Ranking de innovación periodística de la Universidad Miguel Hernández, publicado por primera vez en 2014.

\subsection{Tipologías de la innovación mediática}

La comunidad académica pretende alcanzar una metodología que unifique los criterios de investigación sobre innovación en el mercado mediático, pero que a la vez tenga la suficiente maleabilidad para adaptarse a cada caso concreto. Los expertos en la materia han producido un alto volumen de literatura especializada orientada a estos fines. A continuación, se hará una revisión bibliográfica para revelar qué métodos han sido propuestos y en qué objeto se centra cada uno de los sistemas.

Kamprath y Mietzner (2009) ofrecen una tipología en torno al concepto de innovación tecnológica aplicada al periodismo. Para ellos, existen dos grandes grupos de innovaciones: la innovación en el contenido, que tiene que ver con las "emociones"; y la innovación en tecnología. Esta última se ramifica, por un lado, en tecnologías de transmisión o distribución del producto, y por otro, en tecnologías del contenido, concepto que hace referencia a aquellos recursos aportados por la tecnología que sirven para aumentar el interés del contenido, como las ilustraciones, los efectos visuales, o la ludificación (p. 6).

Eugenia Siapera (2012) propone una clasificación de la innovación periodística que se basa en tres grandes áreas. En primer lugar, la organización, área en la que es posible innovar en lo relativo a convergencia, multitarea y concepción multiplataforma de las empresas. En segundo lugar, el área de los contenidos, que se subdivide en personalización, ludificación e integración de perspectivas múltiples. Por último, el área del público, en la que se clasifica el aumento del consumo de noticias en red, y la consolidación de los "produsuarios", neologismo creado por Axel Bruns (2006) para definir al sujeto consumidor y receptor de información en la Web 2.0. Lindmark et al. (2013), plantean otro modelo distinto, que tiene como peculiaridad la existencia de dos ejes o dimensiones: el tipo de innovación, y el aspecto temporal. En la primera, se diferencian las innovaciones en el contenido (que pueden darse tanto en el mensaje como en la forma), en la manera de consumir el producto, en la producción y distribución de los contenidos, y en el modelo de negocio y la organización de la empresa. En cuanto al aspecto temporal, las innovaciones se clasifican, dependiendo de su madurez, en corto, medio o largo plazo (p.131).

Francis y Bessant (2004) introducen una metodología para el análisis de la innovación en el ámbito del marketing. Los cuatro tipos de innovación reconocidos por estos autores hacen referencia al producto, cuando lo que cambia es el bien o servicio que la empresa ofrece; el proceso, cuando lo novedoso se encuentra en la forma de producir o distribuir el producto; la posición, cuando la innovación afecta a la segmentación del mercado; y la innovación 
paradigmática, cuando se produce una modificación de la filosofía de la empresa o el modelo de negocio (Storsul et al., 2013).

Storsul y Krumsvik (2013) adoptan el patrón de Francis y Bessant para su modelo de clasificación de las innovaciones periodísticas. Sin embargo, lo consideran insuficiente, por lo que le añaden una nueva posibilidad: la innovación social. Este tipo de innovación es descrito por los propios autores como aquella que "entiende las demandas sociales y mejora la vida de la gente" (p. 17).

Por su parte, Bleyen, Lindmark, Ranaivoson y Ballon (2014) establecen una tipología diferente, basada en la clásica distinción importada del ámbito tecnológico, entre innovación en el proceso e innovación en el producto (Schweitzer, 2003; Fagerberg, 2003). Para estos autores, existen cinco categorías: modelo de negocio; producción y distribución; consumo y medio; "inner form", referido a los cambios en el estilo o formato en el que se presentan los contenidos a la audiencia; y "core", que guarda relación con las modificaciones en los mensajes o temas del producto. Según esta clasificación, los dos primeros tipos serían abarcados por la innovación en el proceso, los dos últimos, por la innovación en el producto, mientras que los cambios en el consumo y el medio podrían pertenecer a cualquiera de las dos grandes áreas.

Trappel (2015) establece cinco niveles para aquellos estudios que analicen la innovación en el mercado de la comunicación. En primer lugar, las condiciones estructurales (políticas, económica, legales, etc.), y cómo estas afectan a las iniciativas innovadoras que puedan llevarse a cabo en el mercado. En segundo lugar, la producción de contenido, que tiene relación con las rutinas de trabajo, la ética en las redacciones o la interacción con los usuarios en redes sociales. En tercer lugar, la comunicación y la economía, que se basa en el marketing y en las nuevas formas de obtención de recursos. En cuarto lugar, la distribución de los contenidos a través de canales novedosos. Y por último, la experiencia de usuario, que se centra en retratar los hábitos de consumo de la audiencia y cómo los medios se adaptan a ellos (p. 9-11).

Carvajal et al. (2014) proponen una metodología para estudiar los distintos casos de innovación dentro de las organizaciones periodísticas. Con este modelo, los autores pretenden homogeneizar los criterios de análisis en torno a cuatro grandes áreas de innovación: el producto o servicio; los procesos de producción y/o distribución; la naturaleza de la organización de la empresa; y las acciones de comercialización y/o marketing. Estas cuatro ramas amplias quedan subdivididas en decenas de apartados que sirven de descriptores pormenorizados para aquellas iniciativas que se enmarquen en cualquiera de ellos. Así, por ejemplo, una iniciativa innovadora que tenga como base la red social Twitter, se introducirá en la sección de distribución, y a su vez en el subapartado "redes sociales" (p. 8-13).

El modelo de Carvajal et al. es uno de los más integradores y pormenorizados, pues además de incluir la clasificación por tipo de innovación, también tiene en cuenta el grado de ruptura (incremental o radical), la base (tecnológica propia, tecnológica ajena o no tecnológica), y la finalidad de las innovaciones (penetración de mercado, desarrollo de producto, desarrollo de 
mercado o diversificación). Por ello, fue tenido en cuenta por los investigadores del Grupo de Investigación en Comunicación de la Comunidad Valenciana para la elaboración de su Ranking de la innovación periodística 2014, y además será empleado en el presente trabajo para el análisis de la innovación en los medios de comunicación con base en Estados Unidos.

\section{Metodología}

\subsection{Panel de expertos}

El modelo propuesto por Carvajal et al., y adoptado por el Grupo de Investigación en Comunicación de la Comunidad Valenciana analiza la innovación desde dentro de los propios medios de comunicación. Por ello, es necesario establecer, en primer lugar, un método de selección de casos. Estos casos se corresponderán con aquellos proyectos informativos cuyas iniciativas innovadoras serán halladas y estudiadas posteriormente de manera pormenorizada. Una vez analizadas las iniciativas, se identificarán unas tendencias generales innovadoras en el mercado periodístico estadounidense.

Para determinar qué medios de comunicación eran aptos para ser analizados en el presente estudio, se realizó un sondeo entre expertos en el ámbito del mercado mediático norteamericano, tanto académicos como periodistas y editores. El cuestionario, remitido por correo electrónico a quince expertos, constaba de una sola pregunta: "P1. ¿Cuáles son los proyectos periodísticos más innovadores en Estados Unidos en términos de creación de contenido, marketing, modelos de financiación, organización del trabajo o relación con la audiencia? Puede nombrar diez empresas como máximo". Finalmente, de los quince entrevistados, nueve respondieron a la cuestión: Erin Kissane, Justin Ellis, James T. Hamilton, Jonathan Taplin, Adam Schweigert, Sean Branagan, Amy Eisman, Mathew Ingram y John Pavlik.

\subsection{Criba y selección de casos}

Una vez obtenidos los nombres de los medios de comunicación que, según los expertos, son merecedores de someterse a este análisis, se procede a ordenarlos según diferentes criterios de relevancia. Tras esta jerarquización, se eliminan aquellos casos que menos trascendencia presentan, obteniendo así una muestra definitiva de 10 casos para su posterior examen.

El procedimiento empleado para la criba de medios de comunicación fue tomado del informe del Ranking de innovación periodística 2014, pues se considera que los criterios utilizados por el Grupo de Investigación en Comunicación de la Comunidad Valenciana son los que mejor resumen y cuantifican la relevancia de cada caso concreto. Además, se obtiene una visión poliédrica de la importancia de cada medio, al tener en cuenta variables tanto académicas como a nivel profesional y de usuario. 
Esta metodología de selección parte de tres apartados, ponderados de forma idéntica entre ellos (33\%): a) la fórmula de relevancia social, basada en los datos de los medios en las redes sociales e internet; b) la fórmula de relevancia profesional, que hace referencia a la penetración e interés que cada caso suscita entre los profesionales de la comunicación; y c) la fórmula de relevancia experta, que viene marcada por la cuantificación de los votos emitidos por los expertos previamente referidos. La Figura 1 muestra los porcentajes y datos tenidos en cuenta por el filtro utilizado para cribar los medios que se van a analizar. Todos los datos que constituyen cada una de las fórmulas fueron recogidos en una semana de referencia (semana del 23 de febrero de 2015).

La fórmula de relevancia social se emplea para medir la aceptación del medio entre los usuarios de la red. Se calcula a partir del puesto que ocupa la web del medio en el ranking nacional de Estados Unidos en Alexa, el número de seguidores que tiene cada caso en Twitter, el ratio de retuits (calculado con la tecnología de Twitonomy), y la cifra de seguidores y el ratio de engagement en Facebook (obtenido con la herramienta Likeanalyzer). La ponderación de cada uno de los ítems vendría definido por la siguiente fórmula, aparecida en el mencionado informe: "posición en Alexa [Estados Unidos] (20\%) + seguidores en Twitter (15\%) + ratio RTs $(30 \%)+$ fans Facebook $(10 \%)+$ ratio engagement Facebook $(25 \%)$ ".

La fórmula de relevancia profesional se utiliza para asegurar otra visión de la relevancia de los medios: su trascendencia para los analistas y profesionales de la comunicación. Para llevarla a cabo, se crea un motor de búsqueda en Google en el que se agregan como fuentes los blogs y páginas web de referencia relevantes en el sector del mercado de los medios de comunicación. El motor de búsqueda personalizado de Google funciona de la misma manera que el buscador general, pero solamente actúa sobre los sitios web que el usuario introduzca.

En nuestro caso, las páginas agregadas al motor de búsqueda de Google son PressThink (www.pressthink.org), Buzzmachine (www.buzzmachine.com), Tow Center (www.towcenter.org), The Verge (www.theverge.com), ReCode (www.recode.net), la página personal del periodista y analista mediático Jim Romenesko (www.jimromenesko.com), TechCrunch (www.techcrunch.com), Gigaom (www.gigaom.com), Nieman Lab (www.niemanlab.org), Poynter (www.poynter.org), el blog de Felix Salmon en la web de Reuters (blogs.reuters.com/felix-salmon/) y el blog de The Media Equation en la web de The New York Times (www.nytimes.com/column/the-media-equation).

Una vez creado el motor, se procede a hacer una búsqueda por cada uno de los medios de comunicación en proceso de filtrado. Para la recuperación de resultados, se tienen en cuenta los operadores boleanos AND, OR y NOT, y los términos entrecomillados, mediante lo cual podemos asegurarnos de que obtenemos las entradas adecuadas, y que nuestro trabajo no incurre en fallos por exceso o defecto de información. Una vez realizada la búsqueda, se cuentan los resultados obtenidos, pues este será el indicador que muestre la relevancia profesional de los medios, y que haga posible su cuantificación. 
La fórmula de relevancia experta sirve para conocer cuál es la importancia de cada medio de comunicación para los expertos en el ámbito del mercado comunicativo. Los resultados de esta área se obtienen de manera sencilla, pues el único proceso empleado es la atribución de valor numérico a los votos emitidos por el panel de expertos ya realizado.

Tras obtener todos los datos e introducirlos en una matriz de Microsoft Excel en la que previamente hemos incluido las fórmulas para calcular la puntuación de cada medio, seleccionamos los diez primeros casos. Estos serán los diez medios de comunicación que van a ser objeto de nuestro análisis cualitativo pormenorizado.

Figura 1. Filtro de relevancia mediática. Fuente: elaboración propia. Datos: GICOV

\begin{tabular}{|l|l|l|l|}
\hline \multirow{5}{*}{$\begin{array}{l}\text { Fórmula de relevancia } \\
\text { social (33\%) }\end{array}$} & Alexa (20\%) & \\
\cline { 3 - 4 } & Twitter & Seguidores (15\%) \\
\cline { 3 - 4 } & Fatio RTs (30\%) \\
\cline { 3 - 4 } & $\begin{array}{l}\text { Fórmula de relevancia } \\
\text { profesional (33\%) }\end{array}$ & Resultados en el motor \\
\cline { 2 - 4 } & $\begin{array}{l}\text { Fórmula de relevancia } \\
\text { experta (33\%) }\end{array}$ & $\begin{array}{l}\text { Ratio Engagement } \\
(25 \%)\end{array}$ \\
\hline
\end{tabular}

La Figura 2 presenta los resultados de la fórmula de relevancia mediática aplicada a los casos citados por los expertos. Los datos se muestran clasificados en orden descendente.

Figura 2. Resultado ordenado del filtro de relevancia. Fuente: elaboración propia

\begin{tabular}{|l|r|}
\hline The New York Times & 7,374971405 \\
\hline Buzzfeed & 5,69926105 \\
\hline Vox & 4,633830622 \\
\hline Quartz & 4,361382795 \\
\hline Vice & 3,101197039 \\
\hline ProPublica & 2,890182324 \\
\hline Gawker & 2,479259082 \\
\hline Texas Tribune & 2,258616843 \\
\hline Breaking News & 2,193351081 \\
\hline Circa & 2,16205532 \\
\hline Grantland & 2,141120856 \\
\hline The Guardian & 2,048031044 \\
\hline News Deeply & 1,951948466 \\
\hline Yahoo News Digest & 1,829224163 \\
\hline Los Angeles Times & 1,749368008 \\
\hline
\end{tabular}




\begin{tabular}{|l|r|}
\hline The Atlantic (Atlantic Media) & 1,736977815 \\
\hline Politico & 1,73261822 \\
\hline Medium & 1,633714214 \\
\hline Fivethirtyeight & 1,613347449 \\
\hline WNYC & 1,612165502 \\
\hline Minn Post & 1,590831248 \\
\hline Modern Farmer & 1,386969024 \\
\hline Center for Investigative Reporting & 1,382912885 \\
\hline Ushahidi & 1,312957578 \\
\hline Investigative Reporting Workshop & 1,235786978 \\
\hline Symbolia & 0,6732428074 \\
\hline
\end{tabular}

Según los resultados expuestos anteriormente, los diez medios de comunicación que superaron esta última criba, y cuyas iniciativas innovadoras, por lo tanto, serán estudiadas en el presente trabajo son: Breaking News (NBC), Buzzfeed, Circa News, Gawker, Propublica, Quartz, The New York Times, The Texas Tribune, Vice y Vox.

\subsection{Análisis cualitativo de las iniciativas}

El modelo de Carvajal et al., que se va emplear a continuación, fue adoptado por el Grupo de Investigación en Comunicación de la Comunidad Valenciana para la elaboración del Ranking de innovación periodística 2014 (p. 16-17). No obstante, el presente artículo no pretende cuantificar las iniciativas de cada medio, ya que su principal objetivo no es el de establecer un ranking, como en el citado informe, sino que procura extraer unas tendencias generales de carácter cualitativo. Por ello, la metodología empleada será ligeramente modificada para que se adapte completamente a los objetivos estipulados.

En primer lugar, el análisis desarrollado por el GICOV tenía como objetivo la consecución de un ranking que cuantificara la innovación dentro de cada medio para su posterior clasificación. El presente trabajo no pretende ordenar los medios cuantitativamente, sino identificar las tendencias generales del mercado comunicativo estadounidense. Por ello, se considera conveniente prescindir en nuestra indagación del campo "Grado de innovación", que servía al grupo de investigadores de la Universidad Miguel Hernández para atribuir un valor numérico a cada iniciativa dependiendo de su grado de ruptura con las costumbres mediáticas.

En segundo lugar, se observa que el campo "Finalidad u objetivo de la innovación" no tuvo trascendencia para el resultado final en el informe del Ranking de innovación periodística 2014 tal y como aparece en el modelo propuesto por Carvajal et al. En lugar de subdvidirse el apartado en "penetración de mercado", "desarrollo de producto", "desarrollo de mercado" y "diversificación", en el trabajo del GICOV se utiliza el campo para describir de forma libre el objetivo de la iniciativa innovadora. Se considera que para el presente trabajo será más útil emplear el descriptor de este modo. 


\section{Resultados}

\subsection{Base de la innovación}

En este epígrafe, la presente investigación analizará si la base sobre la que se asientan las iniciativas emprendidas por los medios de comunicación tiene un carácter tecnológico o si, por el contrario, la innovación no está necesariamente asociada a las herramientas tecnológicas. Además, en el caso de identificar una iniciativa como tecnológica, se especificará si los recursos con los que se ha creado pertenecen a la propia empresa (tecnológica propia) o si han sido importados desde otras corporaciones o proyectos (tecnológica ajena). En la Figura 4 se muestra la cuantificación para cada una de las bases de innovación en cada medio, así como los totales correspondientes.

Figura 4. Base de las innovaciones. Fuente: elaboración propia

\begin{tabular}{|l|l|l|l|l|}
\hline Medio & Tecnológica propia & Tecnológica ajena & No tecnológica & Total \\
\hline $\begin{array}{l}\text { Breaking } \\
\text { News }\end{array}$ & 6 & 4 & 3 & 13 \\
\hline Buzzfeed & 8 & 4 & 6 & 17 \\
\hline Circa & 7 & 1 & 3 & 11 \\
\hline Gawker & 5 & 0 & 5 & 10 \\
\hline Propublica & 6 & 5 & 10 & 21 \\
\hline Quartz & 15 & 2 & 10 & 27 \\
\hline $\begin{array}{l}\text { The New York } \\
\text { Times }\end{array}$ & 25 & 2 & 8 & 35 \\
\hline $\begin{array}{l}\text { The Texas } \\
\text { Tribune }\end{array}$ & 10 & 3 & 12 & 25 \\
\hline Vice & 8 & 3 & 8 & 19 \\
\hline Vox & 7 & 1 & 7 & 72 \\
\hline Total & 97 & 25 & & 194 \\
\hline
\end{tabular}

Entre el total de innovaciones con base tecnológica (122), sobresale el grupo de iniciativas cuya base es la tecnología propia, que cuenta con 97 entradas, frente a los 25 registros hallados para el conjunto de las iniciativas fundamentadas en la tecnología de otras empresas. Es decir, que un 79,51\% de las iniciativas tecnológicas se asientan en recursos desarrollados por las mismas empresas que las implementan. Como muestran los datos, los medios de comunicación estadounidenses prefieren diseñar ellos mismos sus propias herramientas para que, de este modo, se amolden completamente a las necesidades, particularidades y estrategias definidas para cada uno de sus proyectos.

Según el Ranking de innovación periodística 2014, entre las iniciativas adoptadas por los medios de comunicación españoles, prevalecen, con un 59,63 \%, aquellas con base tecnológica ajena. Por lo tanto, la actual investigación refleja que las empresas mediáticas estadounidenses están más preparadas y dispuestas para introducir sus propias tecnologías en el mercado, mientras que 
las españolas prefieren aprovechar los recursos habilitados por terceros (redes sociales, GIFs, etc.).

The New York Times es el medio de comunicación que más innova en lo referente a tecnología propia (25 registros), seguido de lejos por Quartz (15) y The Texas Tribune (10). El éxito del diario neoyorquino en esta faceta reside, sobre todo, en la creación de recursos que favorecen la personalización de los contenidos y el engagement de la audiencia. Algunos ejemplos son los buscadores avanzados de artículos, la ludificación por medio de la creación de concursos de preguntas y respuestas sobre actualidad, o la implementación de bases de datos de servicios externos (restaurantes, viviendas para venta y alquiler, u oferta y demanda de trabajo, entre otros). Estas medidas creadas con tecnología desarrollada por el propio medio favorecen la cercanía con la audiencia y la experiencia de consumo de los usuarios.

El informe The New York. Times Innovation Report, elaborado por un equipo de expertos de la empresa norteamericana con el objetivo de impulsar la innovación en el seno de la dama gris, recoge como propuesta de futuro la potenciación, más si cabe, de tecnologías de desarrollo interno para atraer a la audiencia: "Mejorar la tecnología nos proporciona más y mejores herramientas para asegurarnos de que nuestro trabajo llegue a los lectores correctos, en el lugar correcto y en el momento correcto" (p. 26).

En cuanto a las iniciativas con base tecnológica ajena, destaca ProPublica (5), con un pírrico margen sobre Breaking News y Buzzfeed, cuyos registros se sitúan en 4. Las innovaciones con base tecnológica ajena adoptadas por ProPublica se fundamentan en el uso de herramientas para hacer posible el crowdsourcing (recepción de datos o noticias desde fuentes ciudadanas). La habilitación de una cuenta de Whatsapp para recibir información de los ciudadanos, o la petición a las fuentes de que envíen sus noticias al buzón ciudadano Secure Drop Server a través del navegador Tor Browser (que no rastrea la IP de los usuarios) son ejemplos de estas prácticas.

Ya en 2013, Richard Tofel, fundador y director general de ProPublica, comentaba en una entrevista para Nieman Lab la importancia de tener en cuenta a la ciudadanía a la hora de hacer periodismo: "Siempre he soñado con tratar de aprovechar el potencial de la multitud. Es muy desafiante. Hemos hecho algunos experimentos sobre esto. Pienso que estamos encontrando una manera de hacerlo". Como muestran los datos, en 2015 la entidad sin ánimo de lucro continúa avanzando en la búsqueda de canales que exploten la relación con la audiencia. Muchos de estos canales se hacen posible gracias a las plataformas o servicios ofrecidos por terceros.

Las innovaciones sin carácter tecnológico son 72, con lo que suponen un 37,11 \% de las 194 iniciativas anotadas en total. A pesar de quedarse muy por debajo de los datos referidos a iniciativas con base tecnológica (que agregan tecnología propia y ajena), suponen un porcentaje notable. Por ello se deduce que, cuando se habla de innovación en los medios de 
comunicación, cabe desterrar la idea de que esta solamente se produce en el ámbito tecnológico. No siempre es así. De hecho, las iniciativas adoptadas en el terreno no tecnológico pueden llegar a ser más influyentes. A menudo, las innovaciones no tecnológicas consisten en alianzas de producción y distribución, creación de laboratorios tecnológicos y de ideas, o decisiones estratégicas acerca del producto o su comercialización, que son más determinantes para el éxito o fracaso de la empresa.

Entre los medios de comunicación estudiados, el más destacable en materia de innovaciones no tecnológicas es The Texas Tribune, que presenta 12 iniciativas, por delante de ProPublica y Quartz, que cuentan con 10 innovaciones cada uno. Las iniciativas adoptadas por The Texas Tribune e incluidas en este apartado consisten, en su mayoría, en modelos de negocio para financiar su actividad (microdonaciones, contenido patrocinado, etc.), alianzas con otras organizaciones para la creación de contenidos, como el acuerdo firmado con la University of Texas para ofrecer información sobre el mundo estudiantil, o iniciativas en favor de la transparencia y la filosofía open source (fuentes abiertas).

\subsection{Objeto de la innovación}

A continuación, la presente investigación se centra en analizar cuantitativamente el objeto de las innovaciones adoptadas por los medios de comunicación. Las innovaciones se pueden clasificar, según el modelo propuesto por Carvajal et al., en cuatro grupos, que dependen del objeto de las iniciativas: innovaciones propias del producto, del proceso de producción o distribución, de la organización de la empresa, o de la comercialización de los productos o servicios.

En próximos epígrafes, se estudiarán de manera pormenorizada los cuatro apartados, para definir qué iniciativas se adoptan en los medios de comunicación con más frecuencia dentro de cada grupo. En esta parte del trabajo, se ofrecen unas ligeras pinceladas de lo que será expuesto más adelante. Sin embargo, el objetivo principal en este punto es el de averiguar cuáles son los objetos de innovación más comunes. Además, se identifican los medios estadounidenses que más innovan en cada área. En la Figura 5 se encuentran los datos referentes al objeto de las innovaciones en cada medio, así como los totales correspondientes.

Figura 5. Objeto de las innovaciones. Fuente: elaboración propia

\begin{tabular}{|l|l|l|l|l|l|}
\hline Medio & Producto & $\begin{array}{l}\text { Producción/ } \\
\text { Distribución }\end{array}$ & Organización & Comercialización & Total \\
\hline $\begin{array}{l}\text { Breaking } \\
\text { News }\end{array}$ & 4 & 6 & 1 & 2 & 13 \\
\hline Buzzfeed & 5 & 9 & 1 & 3 & 18 \\
\hline Circa & 2 & 6 & 0 & 3 & 11 \\
\hline Gawker & 5 & 4 & 1 & 0 & 10 \\
\hline Propublica & 5 & 10 & 0 & 6 & 21 \\
\hline Quartz & 7 & 5 & 3 & 12 & 27 \\
\hline
\end{tabular}




\begin{tabular}{|l|l|l|l|l|l|}
\hline $\begin{array}{l}\text { The New } \\
\text { York Times }\end{array}$ & 13 & 9 & 6 & 7 & 35 \\
\hline $\begin{array}{l}\text { The Texas } \\
\text { Tribune }\end{array}$ & 4 & 12 & 0 & 9 & 25 \\
\hline Vice & 6 & 6 & 0 & 7 & 19 \\
\hline Vox & 5 & 3 & 1 & 6 & 15 \\
\hline Total & 56 & 70 & 13 & 55 & 194 \\
\hline
\end{tabular}

En Estados Unidos, las innovaciones cuyo objeto es el proceso de producción o distribución suponen el grupo más numeroso, con un 36,08\% del total (70 de 194). Le siguen las áreas de producto, con 56 iniciativas (28,87\% del total), y de comercialización, con 55 innovaciones (28,35 \% del total). Por último, el ámbito de la organización empresarial es el apartado menos abultado, pues cuenta con 13 iniciativas, que conforman el 6,7 \% restante.

Según las iniciativas recogidas en el análisis cualitativo del presente trabajo, la ventaja de los medios estadounidenses en cuanto a innovaciones en el proceso de producción o distribución podrían explicarse atendiendo a tres factores clave. En primer lugar, la abundancia de colaboraciones entre medios de comunicación para la creación, adquisición o distribución de contenidos. En segundo lugar, la proliferación de iniciativas que conciben a la audiencia como algo más que un sujeto pasivo: produsers que, además de recibir la información, la interpretan y hasta la producen. Y en tercer lugar, el auge de las redes sociales, que multiplica las maneras en las que los contenidos llegan al usuario, con lo que se revolucionan los procesos de distribución de contenidos.

En lo referente a la organización empresarial, se observa que el valor es muy reducido en comparación con los demás objetos. Esto podría deberse a que los cambios en la forma de hacer las cosas dentro de las empresas tienen más repercusión para la corporación que innovaciones con otro objeto. Es decir, que son iniciativas poco numerosas pero de una gran envergadura a nivel cualitativo, pues están relacionadas con la filosofía, los valores o la forma de concebir el periodismo y la propia innovación de una corporación. Además, el modelo de análisis propuesto por Carvajal et al. se basa en la observación, por lo cual es complicado identificar innovaciones en la organización, pues estos cambios se producen en sustratos más profundos y menos observables de la empresa.

A continuación, el presente trabajo procede a identificar los medios con las iniciativas más innovadoras en cada uno de los objetos. Estos casos se analizarán con más detalle en los próximos epígrafes, que se centrarán en cada uno de los objetos en concreto.

El medio norteamericano que más innova en su producto es The New York Times, con trece iniciativas encaminadas, como se ha mencionado anteriormente, a incrementar el engagement con la audiencia. Quartz, con siete innovaciones, es el medio que más se aproxima. Este nativo 
digital orienta sus iniciativas en el área del producto a conseguir un diseño de página más limpio, usable, atractivo y moderno, además de historias que, por su formato, tengan más posibilidades de ser leídas por los usuarios.

Sus doce iniciativas en el proceso de producción y distribución hacen que The Texas Tribune se sitúe a la cabeza de la innovación en esa área. Como se ha señalado anteriormente para el medio regional, estas iniciativas corresponden a los acuerdos de producción con colectivos de la sociedad civil, y a la transparencia en la relación con el usuario. The New York Times y Buzzfeed presentan nueve modificaciones en este ámbito. Estas iniciativas se basan en el empleo de las redes sociales para alcanzar a la audiencia, así como al uso de newsletters.

En el área de organización empresarial, el más innovador vuelve a ser el New York Times (seis iniciativas) seguido por Quartz (tres iniciativas). Las modificaciones se centran, sobre todo, en la creación de laboratorios de ideas y nuevas formas de gestionar los procesos de trabajo dentro de las redacciones.

Por último, en el apartado de comercialización, el medio estadounidense más destacable es Quartz, con doce innovaciones. Estas se basan en el cuidado de la apariencia de su sitio web, así como en la diversificación de canales para la obtención de financiación. The Texas Tribune también recurre a la innovación para conseguir los fondos que aseguren su correcto funcionamiento. Por ello, es el segundo medio de comunicación en el área de comercialización, con nueve iniciativas.

En los siguientes epígrafes, la investigación analiza en profundidad cuáles son las principales tendencias innovadores en el mercado americano para cada uno de los objetos de innovación (producto, proceso, organización empresarial y comercialización).

\subsubsection{Innovaciones en el producto}

A continuación, el trabajo presenta un análisis sobre un objeto concreto de la innovación: el producto. Para llevar a cabo esta exposición, se tiene en cuenta la base (tecnológica propia, ajena o no tecnológica) de las iniciativas que se enmarcan en el área del producto. Además, se identifican los conjuntos de ideas con similar naturaleza que más se repiten para determinar cuáles son las tendencias innovadoras en el ámbito del producto con mayor trascendencia en el mercado mediático estadounidense en la actualidad. La Figura 6 recoge los resultados referidos al área del producto, clasificados según su base.

Figura 6. Innovaciones en el producto clasificadas por base. Fuente: propia

\begin{tabular}{|l|l|l|l|l|}
\hline Medio & $\begin{array}{l}\text { Tecnológica } \\
\text { propia }\end{array}$ & $\begin{array}{l}\text { Tecnológica } \\
\text { ajena }\end{array}$ & No tecnológica & Total \\
\hline Breaking News & 2 & 1 & 1 & 4 \\
\hline Buzzfeed & 3 & 1 & 1 & 5 \\
\hline
\end{tabular}




\begin{tabular}{|l|l|l|l|l|}
\hline Circa & 0 & 1 & 1 & 2 \\
\hline Gawker & 3 & 0 & 2 & 5 \\
\hline Propublica & 4 & 1 & 0 & 5 \\
\hline Quartz & 5 & 0 & 2 & 7 \\
\hline The New York Times & 11 & 0 & 2 & 13 \\
\hline The Texas Tribune & 4 & 0 & 0 & 4 \\
\hline Vice & 3 & 2 & 1 & 6 \\
\hline Vox & 3 & 0 & 2 & 5 \\
\hline Total & 38 & 6 & 12 & 56 \\
\hline
\end{tabular}

Se observa que la mayoría de las innovaciones en el producto se basan en tecnología propia de las empresas, con un porcentaje del $66,07 \%$, muy por encima del $21,43 \%$ que presentan las no tecnológicas, y del 10,71\% de las tecnológicas ajenas. Este dato evidencia que los medios de comunicación estadounidenses prefieren servirse de sus propios recursos, tanto tecnológicos como analógicos, a la hora de modificar su producto.

Entre las innovaciones que tienen el producto como objeto, se aprecia una presencia importante de iniciativas centradas en la gestión de los comentarios vertidos por los usuarios en los artículos del medio, ya sea por el lugar en el que se sitúan, por el espacio que ocupan o por la importancia que se les otorga. Se percibe, por tanto, que los medios digitales más innovadores apuestan por implementar cambios destinados a reconocer y aprovechar la gran relevancia de la audiencia para el nuevo sistema mediático.

En este sentido, es especialmente revolucionario el sistema de comentarios empleado por Gawker, Kinja, en el que se introducen cambios constantemente. La tecnología, lanzada por la propia empresa en 2012, concede al usuario un papel importante dentro del medio, ya que le permite crear su propio blog dentro de la red de Kinja. En este espacio, la audiencia podrá publicar sus propias historias, se guardarán los comentarios que haga en otros blog de la red, y podrá mantener conversaciones tanto con los periodistas como con otros usuarios de igual a igual. El dueño de Gawker, Nick Denton, aseguraba en una entrevista para ReCode que la interacción con los usuarios es una "condición previa" para que las empresas mediáticas digitales puedan alcanzar el éxito.

El sistema de comentarios utilizado por Quartz también merece una especial atención por su carácter innovador. Este medio de comunicación estadounidense sitúa un botón a la derecha de cada párrafo en sus artículos. Presionando este botón, el usuario puede introducir comentarios, que se guardan como anotaciones a la derecha del párrafo seleccionado. Mediante este sistema, se cambia sustancialmente el modo de comentar los artículos, pues la audiencia ya no tiene que esperar al final para ofrecer sus puntos de vista, sino que puede hacerlo justo al lado del contenido o dato publicado por el medio de comunicación. 
La ludificación de los contenidos sirve también para aumentar la interacción con el usuario y conseguir así su fidelización. Es decir, introducir a los contenidos un componente de reto hace que la audiencia juegue y se divierta. Uno de los máximos valedores de estas innovaciones es Buzzfeed, que dedica una sección entera, Quizzes, a cuestionarios y concursos.

El periodismo explicativo también es una nueva tendencia entre las innovaciones adoptadas en el ámbito del producto. La tecnología permite a los medios de comunicación utilizar cada vez más mapas, gráficos e infografías para explicar de la forma más sencilla posible datos y realidades complejas. En este contexto destacan iniciativas como la de The Texas Tribune, consistente en la creación del apartado Tribpedia dentro de la web del medio. Esta sección sirve como enciclopedia de actualidad, en la que el usuario puede consultar, por ejemplo, quién es cualquier personaje aparecido en informaciones del Texas Tribune.

The Upshot es el blog de periodismo explicativo de The New York Times. Nacido bajo el paraguas del gigante mediático norteamericano, el sitio cubre la ausencia del pionero en la especialidad, FiveThirtyEight, que pertenecía a la edición digital de la dama gris desde agosto de 2010, pero fue adquirido y relanzado por la ESPN entre finales del año 2013 y principios de 2014.

Vox es un medio de comunicación enteramente dedicado al periodismo explicativo. La empresa propietaria de este medio, Vox Media, lo define en su página web como "el sitio al que se puede ir para entender las noticias y el mundo que nos rodea". De hecho, el medio cuenta con una sección llamada Explainers, en la que los periodistas de Vox exponen de forma sencilla y esquematizada aquellos temas de actualidad que presentan cierto nivel de complejidad para el lector medio.

La adaptación de los contenidos al formato digital y a los hábitos de consumo de la audiencia son preocupaciones latentes para los medios de comunicación innovadores. Las iniciativas que buscan mejorar el producto ofrecido en este sentido son abundantes. Mediante la jerarquización de la información, los buscadores avanzados o la fragmentación de las historias, se facilita la navegación del usuario a través de la página web del medio. Además, todos los sitios web estudiados son adaptables a dispositivos móviles, lo cual facilita en gran medida la navegación.

Circa presenta uno de los formatos más innovadores de todos los medios analizados. Además de emplear los dispositivos móviles como su plataforma nativa, todas sus historias se lanzan a la audiencia fragmentadas, lo que da lugar a una nueva forma de leer y entender las noticias.

Breaking News destaca también en cuanto al formato de presentación de contenidos, pues el medio consiste en un feed infinito de microposts (similar a la red social Twitter). En él se van publicando al segundo informaciones que provienen de periodistas de la NBC, dueña de Breaking News, y de diversas agencias de noticias. 
La consolidación del streaming informativo y el podcast también se hacen evidentes en este grupo de iniciativas tecnológicas propias de producto. En el caso del streaming, se empieza a introducir en ámbitos locales (como hace el Texas Tribune con su sección Livestream), pero sobre todo, se desarrolla su reproducción en aplicaciones para teléfonos inteligentes. La NBC ya ofrece vídeo en directo de los eventos más importantes a través de la aplicación de Breaking News para iOS.

La utilización del vídeo en las informaciones digitales es un hecho afianzado incluso para los medios de comunicación más tradicionales. Sin embargo, el formato evoluciona en algunos casos para ofrecer un producto más innovador y útil para la audiencia. Es el caso de Vox, que produce vídeos con infografías integradas. Por ejemplo, en la segunda semana de febrero de 2015, el medio publicó una entrevista con el presidente de los Estados Unidos, Barack Obama, en la que van apareciendo datos, gráficos, y otros tipos de piezas infográficas que ilustran las palabras del mandatario.

\subsubsection{Innovaciones en el proceso de producción o distribución}

En este punto del análisis, el presente trabajo desgrana las claves de la innovación fundamentada en los procesos de producción y distribución del producto. La Figura 7 muestra los datos correspondientes a las iniciativas innovadoras cuyo objeto es el proceso. Estos resultados están clasificados, además, según su base.

Figura 7. Innovaciones en los procesos clasificadas por base. Fuente: propia

\begin{tabular}{|l|l|l|l|l|}
\hline Medio & $\begin{array}{l}\text { Tecnológica } \\
\text { propia }\end{array}$ & $\begin{array}{l}\text { Tecnológica } \\
\text { ajena }\end{array}$ & No tecnológica & Total \\
\hline Breaking News & 4 & 2 & 0 & 6 \\
\hline Buzzfeed & 5 & 3 & 1 & 9 \\
\hline Circa & 5 & 0 & 1 & 6 \\
\hline Gawker & 2 & 0 & 2 & 4 \\
\hline Propublica & 0 & 4 & 6 & 10 \\
\hline Quartz & 1 & 1 & 3 & 5 \\
\hline The New York Times & 6 & 2 & 1 & 9 \\
\hline The Texas Tribune & 2 & 3 & 7 & 12 \\
\hline Vice & 2 & 1 & 3 & 6 \\
\hline Vox & 1 & 1 & 1 & 3 \\
\hline Total & 28 & 17 & 25 & 70 \\
\hline
\end{tabular}

En estos datos se puede observar que, a pesar de que las innovaciones tienen en su mayoría una base tecnológica propia (40 \%), el grupo de las iniciativas no tecnológicas no se queda atrás $(35,71 \%)$. El apartado que menos innovaciones presenta es el de la base tecnológica ajena, aunque se observa que el registro no es bajo y que, de hecho, el objeto que más innovaciones tecnológicas ajenas presenta es el de los procesos de producción y distribución. 
De entre las innovaciones tecnológicas propias, tienen una presencia notable (25\% de las 28 iniciativas) aquellas referidas al envío de newsletters a los usuarios que se suscriban para ello. Se pretende con esto fidelizar a la audiencia, ofrecer un seguimiento de los temas más relevantes, y aprovechar absolutamente todas las plataformas para la publicación de los contenidos del medio. Además, en la mayoría de los medios existe la posibilidad de suscribirse a una sección solamente. Por ejemplo, The New York Times permite recibir en el correo electrónico historias de la sección de cocina únicamente. Buzzfeed, por su parte, cuenta con 16 secciones diferentes que envían newsletter a los suscriptores.

El Brief de Quartz es un exponente relevante de newsletter que consiste en un resumen de actualidad que se envía a los suscriptores a primera hora de la mañana. Gracias a su éxito, influyó en el rediseño de la homepage del medio, en agosto de 2014. Gideon Lichfield, editor de Quartz, afirmaba en junio de 2014 en The New York Times que otro medios han rechazado el email como "algo que la gente antigua usa". Sin embargo, aseguraba que "en los últimos años, hemos empezado a ver el correo electrónico como algo igual a plataformas de publicación, como Twitter, Facebook y la web".

El Quartz Global Executive Study, un informe elaborado por el equipo de mercadotecnia de la empresa, titula uno de sus epígrafes con la siguiente frase: "El buzón de entrada del correo electrónico es la nueva homepage para los ejecutivos". La afirmación se asienta en el dato de que el $60 \%$ de este segmento de población consulta el correo electrónico como una de sus tres fuentes de noticias diarias, muy por encima del $46 \%$ que supone el navegador web y las redes sociales, y el $28 \%$ de las aplicaciones de noticias.

Los datos reflejan un número abultado de iniciativas no tecnológicas dentro del área de los procesos de producción y distribución. Las tendencias mediáticas que lo provocan son las alianzas de producción y distribución con otras empresas, colectivos y plataformas; la transparencia empresarial y laboral dentro de los propios medios; y la promoción de la filosofía open data (datos libres y a disposición del usuario).

Las alianzas de producción y distribución constituyen una de las tendencias más relevantes tanto en la innovación mediática estadounidense. Estas iniciativas permiten a los medios aunar recursos y esfuerzos para conseguir metas que de otra manera serían inalcanzables. La alianza múltiple entre Mashable, Quartz, Mother Jones, Digg y Breaking News para la creación de Ukraine Desk, una cuenta de Twitter generada para informar sobre la situación política en Ucrania durante las revueltas del Euromaidan, es un ejemplo claro de este tipo de innovaciones.

Estas innovaciones también permiten aumentar la exclusividad del producto. La alianza entre Samsung y Vice tiene como objetivo que los vídeos editados del programa en streaming On the line, emitido por el medio norteamericano, solo puedan ser reproducidos en dispositivos de dicha marca. 
The Texas Tribune es un referente en cuanto a la adopción de acuerdos con otras entidades para ofrecer al ciudadano servicios útiles. Su colaboración con Southwestern Medical Center para publicar información sanitaria en la sección Trib + Health, el acuerdo con la University of Texas para la creación del espacio Trib+Edu, donde se comunican noticias del ámbito estudiantil, y la estrecha relación con el diario The Washington Post para la cofinanciación de charlas y eventos, son algunos de los ejemplos más relevantes.

En lo referente a la transparencia, se observa que son muchos los medios de comunicación estudiados que incluyen un apartado de fe de erratas en el que se muestran los errores cometidos por los periodistas, incluso en la edición digital. Una de las empresas que más énfasis pone en este sentido es el New York Times, que busca mantener el rigor de su cabecera. Estas iniciativas no suponen grandes cambios tecnológicos ni de infraestructura, pues el concepto es bastante simple. Sin embargo, es impensable que los medios de comunicación españoles, sobre todo los tradicionales, evidencien sus propios errores y publiquen cuándo y por qué se corrigen los artículos de su edición digital.

Existen iniciativas innovadoras relacionadas con la transparencia que son incluso más comprometidas. En enero de 2015 surgió un conato de conflicto en la redacción de Gawker entre los directivos y los trabajadores de Jezebel, el espacio del medio dedicado a la mujer. El blog The Awl, editado por el ex editor de Gawker Choire Sicha, filtró un plano de la redacción donde se podía ver qué escritorio pertenece a cada periodista. Según los trabajadores, el gráfico filtrado en favor de la transparencia podría poner en peligro la seguridad de la plantilla después de los atentados en la revista francesa Charlie Hebdo.

The Texas Tribune es uno de los medios que más destacan en el ámbito de la transparencia. El diario digital publica en su página web el nombre de todos sus anunciantes y mecenas, y presenta, en la sección de Donantes y miembros, el estado actualizado de sus cuentas.

También es habitual que los medios de comunicación innovadores en Estados Unidos expliquen con naturalidad las modificaciones sufridas por su producto cuando se hacen rediseños de sus páginas web. La aplicación Circa tiene un espacio en la red de blogs Medium destinada, únicamente, a presentar los cambios en su medio de comunicación, así como las razones que los motivan. Gawker y Quartz también innovan en este aspecto, pues ambos medios de comunicación publicaron sendos artículos tras sus respectivos rediseños para explicar a su audiencia los motivos del cambio.

En relación con los puntos anteriormente expuestos, los medios innovadores suelen promover la apertura de datos (open data), y de códigos de programación (open source). Es decir, que abogan por compartir sus recursos. Por ejemplo, ProPublica tiene la sección Steal Our Stories, donde se permite que los usuarios republiquen los artículos, siempre y cuando se cite que proceden de este medio. En este sentido, son más abundantes las innovaciones no 
tecnológicas, pues lo realmente rompedor es el concepto de compartir y no la forma en que se hace. Además, medios como The New York Times, Quartz o Propublica emplean la tecnología ajena, como la plataforma Github, para compartir sus repositorios.

Quartz es un ejemplo altamente destacable de empresa que favorece la filosofía open source, pues pone a disposición de la audiencia la herramienta para crear gráficos Chartbuilder, un software creado por los desarrolladores del propio medio de comunicación norteamericano.

En cuanto a las iniciativas tecnológicas ajenas, las innovaciones que más presencia tienen están relacionadas con la creación, por parte de los medios de comunicación, de perfiles en redes sociales que no son habituales para la difusión de noticias. Es decir, que las empresas no se limitan a publicar en Twitter y Facebook, sino que muchas de ellas mantienen actualizado su Pinterest, Instagram, Tumblr o Google +.

Es llamativo el caso de The Texas Tribune. El medio regional tiene una cuenta en Instapaper, una red social que permite compartir artículos y contenidos periodísticos con tus seguidores. Esta plataforma todavía cuenta con muy poca implantación entre las empresas mediáticas y la audiencia.

Las cuentas de The New York Times en Instagram y Tumblr son especialmente interesantes. El medio norteamericano presta mucha atención a estas redes sociales, en las que publica imágenes de gran valor plástico e informativo, y aprovecha el espacio destinado al texto para contextualizarlas con historias cuidadas y llamativas, que enganchen a los usuarios de la plataforma.

Snapchat se ha convertido recientemente en una red social en la que los medios de comunicación quieren publicar sus contenidos, dejando atrás la idea de que la plataforma servía únicamente a un público adolescente. El diario The New York Times también es un caso a destacar en este aspecto, pues utiliza la aplicación para crear historias nativas, y ya está planificando la cobertura de las elecciones de 2016 a través de dicha plataforma.

Facebook ha sabido percibir la creciente dependencia de los medios de comunicación en las redes sociales, por lo cual ha lanzado Instant Articles en colaboración con diversas empresas mediáticas, entre las que se encuentran Buzzfeed y The New York Times. Instant Articles es una herramienta incorporada a la aplicación de Facebook para dispositivos móviles que permite a los publicadores ofrecer sus artículos de manera más rápida e interactiva. Los contenidos se cargarán de la misma manera que en la actualidad lo hacen las fotos y vídeos, por lo que el tiempo de espera será menor, y además, se ofrecerán características complementarias, como cápsulas de sonido para que el autor explique un artículo. 


\subsubsection{Innovaciones en la organización empresarial}

En este epígrafe, la investigación se centra en el objeto de las innovaciones de la organización empresarial. Todas las innovaciones aquí presentes estarán asociadas a las modificaciones de la estructura interna de las empresas. La Figura 8 muestra los datos correspondientes a esta área de la innovación clasificada también por su base.

Figura 8. Innovaciones en la organización clasificadas por base. Fuente: propia

\begin{tabular}{|l|l|l|l|l|}
\hline Medio & $\begin{array}{l}\text { Tecnológica } \\
\text { propia }\end{array}$ & $\begin{array}{l}\text { Tecnológica } \\
\text { ajena }\end{array}$ & No tecnológica & Total \\
\hline Breaking News & 0 & 0 & 1 & 1 \\
\hline Buzzfeed & 0 & 0 & 1 & 1 \\
\hline Circa & 0 & 0 & 0 & 0 \\
\hline Gawker & 0 & 0 & 1 & 1 \\
\hline Propublica & 0 & 0 & 0 & 0 \\
\hline Quartz & 1 & 1 & 1 & 3 \\
\hline The New York Times & 4 & 0 & 2 & 6 \\
\hline The Texas Tribune & 0 & 0 & 0 & 0 \\
\hline Vice & 0 & 0 & 0 & 0 \\
\hline Vox & 0 & 0 & 1 & 1 \\
\hline Total & 5 & 1 & 7 & 13 \\
\hline
\end{tabular}

A primera vista, la presente investigación revela que el grupo más numeroso de innovaciones en lo referente a la organización empresarial es el de base no tecnológica, seguido por el de base tecnológica propia y, a continuación, el de base tecnológica ajena. Sin entrar a pormenorizar cuáles son las tendencias más rompedoras en esta área, se percibe que las iniciativas no tecnológicas guardan relación con la organización del personal dentro de la redacción; las tecnológicas propias, con la creación de laboratorios de experimentación; y la tecnológica ajena, con herramientas externas para la gestión de la información en las plantillas.

La incorporación de desarrolladores, programadores y diseñadores las redacciones o, en su defecto, la contratación de periodistas que tengan un alto nivel de conocimiento en estas materias es una tendencia innovadora especialmente relevante, en lo referente al ámbito no tecnológico en materia de organización empresarial.

Medios como Buzzfeed presentan rutinas de trabajo diferentes de las tradicionales, en las que los periodistas se dedicaban únicamente a un formato, ya fuera audio, vídeo, escrito o digital. Por el contrario, en estas redacciones se cuenta con periodistas capaces de elaborar todo tipo de informaciones, pues tienen conocimientos suficientes sobre las técnicas y tecnologías necesarias en todos los procesos productivos.

La innovación mediática se persigue como una obsesión en la cúpula directiva de las empresas, por lo que es cada vez más común que se creen cargos con el objetivo de promoverla. En este sentido, The New York Times ha introducido el puesto del Innovation Editor, que asegura la 
constante renovación de sus formatos y contenidos. Cuando se confirmó la toma de posesión de Kinsey Wilson en este puesto, en noviembre de 2014, Dean Banquet, director ejecutivo del Times, aseguraba que el medio de comunicación se encuentra "en una época crucial, al tiempo que aceleramos nuestra transformación en una organización periodística que distribuye periodismo de calidad en todas las plataformas".

The New York Times ha implementado también una iniciativa para modernizar los procesos internos para la toma de decisiones. Esta innovación es la adopción de las Dean's Lists, dos listas de noticias digitalizadas que ayudan a jerarquizar las informaciones en la web del medio. Esta iniciativa agiliza el proceso de elegir qué temas aparecerán en la portada de la siguiente edición, suprimiendo con ello las tradicionales reuniones en la redacción, más propias de una época predigital.

Los medios de comunicación crean laboratorios internos con tecnología propia para experimentar con nuevos formatos y decidir qué iniciativas se podrían incorporar al producto final. Destaca en este sentido el diario The New York Times, que tiene una red de laboratorios entre los que se encuentran Idea Lab, Beta620 o R\&D Lab.

Quartz también es innovador en este sentido, pues este medio de comunicación creó Glass, un laboratorio de la televisión y los formatos audiovisuales que, aunque en la actualidad no publica contenidos en su blog original por decisión empresarial, continúa su actividad en su perfil de Twitter.

Buzzfeed ha anunciado que a finales del verano de 2015 empezará a funcionar el Open Lab for Journalism Technology and the Arts. Este laboratorio tiene como objetivo "lanzar nuevas herramientas y tecnologías - tanto software como hardware - que beneficien al reporterismo y al periodismo", según han explicado en un artículo publicado en la web de Buzzfeed el 27 de mayo Mat Honan, jefe de la oficina de la compañía en San Francisco, y Johan Peretti, fundador de la corporación. Esta innovación no se recoge en la presente investigación, pues se implementará fuera del período de análisis. Sin embargo, se considera relevante que una de las compañías punteras en el ámbito digital cree su propio laboratorio de recursos para la innovación.

En el grupo de las innovaciones de tecnología ajena, se encuentra la utilización por parte de Quartz de Slack, una herramienta que ayuda a gestionar las plantillas de trabajadores, pues organiza la redacción y contribuye a solucionar problemas que puedan surgir en grupos de trabajadores. Esta aplicación web innova en la forma en la que los trabajadores se comunican e interactúan entre ellos. 


\subsubsection{Innovaciones en la comercialización}

A continuación, el presente trabajo analiza las innovaciones que tienen como objeto la comercialización. Este término debe entenderse en sentido amplio, pues en esta área se estudian todas aquellas iniciativas que contribuyen a la financiación de las empresas que las implementan, ya sea con la comercialización directa del producto, como con cualquier otra estrategia empresarial para conseguir ingresos o posicionar su marca. La Figura 9 muestra las iniciativas correspondientes a este objeto de innovación. Además, se clasifican también dependiendo de su base.

Figura 9. Innovaciones en la comercialización clasificadas por base. Fuente: propia

\begin{tabular}{|l|l|l|l|l|}
\hline Medio & $\begin{array}{l}\text { Tecnológica } \\
\text { propia }\end{array}$ & $\begin{array}{l}\text { Tecnológica } \\
\text { ajena }\end{array}$ & No tecnológica & Total \\
\hline Breaking News & 0 & 1 & 1 & 2 \\
\hline Buzzfeed & 0 & 0 & 3 & 3 \\
\hline Circa & 2 & 0 & 1 & 3 \\
\hline Gawker & 0 & 0 & 0 & 0 \\
\hline Propublica & 2 & 0 & 4 & 6 \\
\hline Quartz & 8 & 0 & 4 & 12 \\
\hline The New York Times & 4 & 0 & 3 & 7 \\
\hline The Texas Tribune & 4 & 0 & 5 & 9 \\
\hline Vice & 3 & 0 & 4 & 7 \\
\hline Vox & 3 & 0 & 3 & 6 \\
\hline Total & 26 & 1 & 28 & 55 \\
\hline
\end{tabular}

Según los datos ofrecidos por la investigación para el área de comercialización, la mayoría de las innovaciones tienen base tecnológica propia y no tecnológica. A la hora de innovar, la mayoría de las iniciativas se fundamentan en acuerdos con anunciantes, cambios en la forma de patrocinio o actividades al margen de la web. Sin embargo, cuando se adoptan cambios en el contexto digital, la mayor parte de las modificaciones surgen de los recursos tecnológicos del propio medio, y no de otras empresas.

Anteriormente, la presente investigación identificaba las newsletters como procesos de distribución en los cuales las empresas comunicativas estadounidenses innovan en la actualidad. Además de esto, estos correos electrónicos también sirven como fuentes de ingresos, pues a menudo son patrocinados por alguna entidad o marca. Medios como Vox o Vice utilizan esta vía de ingresos para conseguir más financiación.

La publicación de contenido patrocinado por otras empresas y entidades es una tendencia creciente entre los medios de comunicación norteamericanos en la búsqueda de formas de publicidad no invasivas. Por ejemplo, la entidad financiera Discover patrocina una sección completa en Vox. Otro ejemplo es la reproducción de música en el blog Noisey, de Vice, que está patrocinada por la conocida marca de snacks Doritos. 
La investigación revela los esfuerzos de algunas de las empresas por implementar una publicidad nativa, tratando de dejar atrás la antigua tendencia digital de los banners y los anuncios estáticos. Vice también destaca en este sentido, pues ha creado el laboratorio para la publicidad AdVice Networks. En este espacio, los diseñadores y programadores del medio proponen diferentes modelos de anuncios comerciales para que los usuarios puedan interactuar con ellos y valorar su nivel de eficacia y utilidad.

Buzzfeed centra sus esfuerzos por encontrar una publicidad nativa y poco invasiva en el product placement. Es decir, que incluye a las marcas patrocinadoras como información de contexto en algunos de los contenidos que se publican en su página web. Jonathan Perelman, vicepresidente de Buzzfeed Motion Pictures explicaba en una entrevista para el portal AdWeek que "si se produce un buen contenido, no solo va a dar igual que se incluya un anuncio, sino que se apreciará como marca comercial".

El crowdfunding y las donaciones de particulares y colectivos son otra forma de obtener ingresos para los medios de comunicación innovadores en Estados Unidos. The Texas Tribune y Propublica, los dos medios sin ánimo de lucro estudiados, se financian a través de este tipo de aportaciones. La causa principal detrás de este dato es que ambos medios sirven como instrumentos de control para evitar abusos por parte de los poderes públicos y las grandes corporaciones. Mediante este sistema de financiación, los diarios se aseguran la independencia de su línea editorial, y así seguir trabajando por el ciudadano.

El micromecenazgo es una variante de este tipo de financiación. Se utiliza de forma extendida en las empresas mediáticas americanas, y consiste en donaciones que se realizan con un objetivo concreto (un proyecto, una adquisición, etc.) y no por el mero hecho de ayudar a la supervivencia del medio de comunicación.

La organización de eventos y charlas con miembros relevantes de la sociedad civil es otra forma de conseguir ingresos, pero sobre todo, es una buena manera para posicionar la marca de la cabecera que los promueve y coordina. The Texas Tribune y el Washington Post colaboran entre sí para la cofinanciación de eventos que ofrezcan un servicio a la comunidad, y con ello mejoren la relación entre su marca y su audiencia.

Aunque no puede ser considerado un evento en el sentido estricto de la palabra, el diario The New York Times organiza viajes que pueden ser contratados por los lectores. Aquellas personas que lo deseen pueden desplazarse a diferentes destinos alrededor de todo el mundo acompañadas por periodistas de The New York Times. Estos profesionales son conocedores de la cultura autóctona de los lugares que se visitan, por lo que ayudan a los turistas a comprender sus costumbres y su historia. 
La viralidad de los contenidos es una estrategia efectiva para el posicionamiento de las marcas en el mercado. Buzzfeed es el medio de comunicación que mejor ha sabido explotar este tipo de iniciativas en el mercado estadounidense. En esta empresa, los contenidos están completamente orientados a conseguir una tasa elevada de publicación en redes sociales. Buzzfeed acepta la publicación en su web de contenidos que generen emociones en la audiencia aunque no cumplan una función informativa esencial. Además, los redactores del medio escriben los titulares como si fueran a ser publicados automáticamente como tuits en la red social Twitter. La estructuración de los datos e historias que componen cada artículo en listas enumeradas contribuye también a que los contenidos se hagan virales.

La apuesta editorial de un medio es una seña de identidad esencial a la hora de posicionar su marca, pues esta es su orientación en la búsqueda de un nicho que permita al medio dirigir su producto hacia un cierto sector de la población. Esta segmentación se puede producir con respecto a los intereses de la audiencia, o a raíz de las características demográficas de la misma. Entre los medios de comunicación estudiados, se encuentran sobre todo medios con apuesta editorial demográfica. Por ejemplo, Vice y Buzzfeed tienen una línea editorial destinada a la población joven. En el caso de Vice, sobre todo a los jóvenes más subversivos y críticos con el sistema. Quartz también cuenta con una apuesta editorial destacable, pues sus contenidos están destinados a ejecutivos o personas relacionadas con el ámbito de los negocios.

En la actualidad, la aplicación de noticias Circa no está obteniendo los resultados esperados tras su lanzamiento en 2013. Frédéric Filloux aseguraba en un artículo para la web sobre medios, tecnología y modelos de negocio Monday Note en mayo de 2015, que una de las causas de este "fiasco" es la indefinición editorial del medio: "la diferenciación editorial es un factor clave. Y Circa no la tenía. Un buen continente es una cosa, pero no puede mantener a un medio sin la ayuda de una línea editorial original, específica e identificable". Se puede apreciar aquí la importancia de tener una apuesta editorial definida como estrategia de posicionamiento de la marca.

Por último, la prestación de servicios no relacionados con la comunicación también sirve como fuente de ingresos y como estrategia de posicionamiento de marca. Por ejemplo, el diario The New York Times ofrece servicios inmobiliarios por los que obtiene dinero. Además, con estas acciones, la marca también adquiere una nueva dimensión, y aquellos usuarios que los utilicen, intensificarán su relación con el medio.

\section{Conclusiones}

A raíz de los resultados anteriormente expuestos, la investigación aporta una serie de claves que permiten entender cómo está evolucionando la innovación en el mercado mediático estadounidense. 
En primer lugar, los medios de comunicación de Estados Unidos emplean tecnología propia a la hora de innovar. Por ello, en estas empresas los periodistas trabajan en redacciones integradas junto con desarrolladores y programadores.

En segundo lugar, medios tradicionales como The New York Times se colocan a la cabeza de la innovación en Estados Unidos, creando laboratorios y apostando fuerte por implementar modificaciones significativas. Mientras tanto, en España, los medios tradicionales llegan tarde incluso a la convergencia digital.

En tercer lugar, el contenido generado por la audiencia (produsers) es una materia a tener en cuenta para los medios innovadores estadounidenses, pues una correcta gestión del mismo puede traducirse en un aumento del engagement y la interactividad.

En cuarto lugar, la ludificación de los contenidos es una innovación que contribuye a mejorar la relación de la audiencia con la cabecera del medio.

En quinto lugar, se percibe la adopción de innovaciones que permitan el desarrollo del periodismo explicativo por parte de algunos medios como Propublica, The Texas Tribune o Vox. Este consiste en la utilización de recursos infográficos para mejorar la comprensión de la actualidad y la realidad que nos rodea.

En sexto lugar, se observa que los medios de comunicación más innovadores aprovechan las posibilidades del formato digital para estructurar los contenidos de acuerdo con los ritmos acelerados de la actualidad. Además, el diseño de todas las páginas web es adaptable a todos los dispositivos.

En séptimo lugar, la investigación muestra la proliferación del streaming y el podcast, incluso en dispositivos móviles.

En octavo lugar, los medios de comunicación mezclan los diferentes formatos existentes para crear nuevas formas de periodismo, aprovechando las posibilidades digitales en favor de una mayor comprensión de las noticias, como hizo Vox en la entrevista a Barack Obama.

En noveno lugar, las newsletters son utilizadas en Estados Unidos de forma altamente extendida, pues la mayoría de los medios de comunicación cuentan con uno o varios resúmenes que se envían periódicamente a la audiencia suscrita a estos fines. Además de conseguir aumentar el tráfico en la página web del medio, estas iniciativas significan un patrocinio diferente que permite abrir una nueva vía de financiación.

En décimo lugar, las empresas emplean la transparencia como vehículo para hacer que la audiencia sienta al medio de comunicación de manera más cercana. Empresas como The Texas Tribune o Propublica marcan la pauta en este sentido.

En undécimo lugar, debido a la escasez de recursos con la que cuentan la mayoría de las empresas mediáticas en la actualidad, los medios adoptan acuerdos de producción y/o distribución. Mediante estas alianzas, los medios de comunicación pueden hacer llegar a los ciudadanos informaciones que de otra manera no sería posible.

En duodécimo lugar, el presente estudio revela que los medios estadounidenses innovadores están abiertos a compartir contenidos y datos (open data), así como recursos de programación (open source).

En decimotercer lugar, los medios de comunicación estadounidenses utilizan redes sociales que no suelen ser habituales para empresas de noticias. 
En decimocuarto lugar, en Estados Unidos los medios crean cargos directivos y asumen procesos de organización de las redacciones que agilizan la toma de decisiones y fomentan la innovación, así como la integración en el ecosistema digital.

En decimoquinto lugar, es frecuente para los medios norteamericanos innovadores con más recursos la creación de laboratorios para explorar nuevos formatos, narrativas rompedoras e incluso formas de publicidad nativa.

En decimosexto lugar, la mayoría de los medios de comunicación innovadores tiene una línea editorial marcada, que les permite posicionarse con éxito en el mercado. Los medios que carecen de esta línea tienen muy difícil encontrar su público objetivo para sobrevivir. En el caso de Estados Unidos, encontramos un ejemplo en la aplicación Circa.

En decimoséptimo lugar, algunos medios de comunicación de Estados Unidos, como Buzzfeed, conceden gran importancia a la viralidad de sus contenidos. Su estrategia de redacción de las informaciones es la previsión del efecto que tendrán las publicaciones en redes sociales.

En decimoctavo lugar, la publicidad nativa y no invasiva es una de las preocupaciones más frecuentes de los medios de comunicación estadounidenses en lo relativo a la financiación. El product placement, los anuncios interactivos y el contenido patrocinado son algunos de los sistemas empleados por las empresas.

En decimonoveno lugar, las donaciones y micromecenazgos sirven a los medios estadounidenses para la financiación de proyectos manteniendo una línea independiente del poder político y económico.

En vigésimo lugar, las empresas mediáticas norteamericanas organizan eventos y charlas con personajes relevantes para posicionar su marca.

\section{Bibliografía}

Asociación de Editores de Diarios Españoles (2015): Libro blanco de la prensa diaria. Recuperado el 15 de abril de 2015 de http://www.aede.es/nota-de-prensa/libro-blanco- de-la-prensadiaria-6/

Bleyen, V.A., Lindmark, S., Ranaivoson, H., Ballon, P. (2014): "A typology of media innovations: Insights from an exploratory study". The Journal of Media Innovations 1(1), Noruega, pp. 28-51.

Bruns, Axel (2006): "Towards Produsage: Futures for User-Led Content Production" en VVAA, Cultural Attitudes towards Communication and Technology 2006 (Coords. Sudweeks, Fay, Hrachovec, Herbert, Ess y Charles). Tartu, Estonia.

Carvajal, M. (2012): "Estrategias de distribución del contenido periodístico en dispositivos móviles. Análisis comparativo de los principales editores de prensa española”, en VVAA, Actas - IV Congreso Internacional Latina de Comunicación Social. Tenerife: Universidad de La Laguna.$$
\text { y Arias, F., Negredo, S., }
$$

Amoedo, A. (2014). "Aproximación metodológica al estudio de la innovación en periodismo". Observatorio. Lisboa: Obercom

Dogruel, L. (2014): "What is so Special about Media Innovations? A Characterization of the Field". The Journal of Media Innovations 1(1), Noruega, pp. 52-69. 
Drucker, P. (1995): "Managing in a Time of Great Change". Harvard Business Press.

Fagerberg, J. (2004): "Innovation. A Guide to Literature", en VVAA, The Oxford Handbook of Innovation (Coords. J. Fagerberg, D. C. Mowery, R. R. Nelson). Oxford: Oxford University Press.

Filloux, F. (2015): “Circa: What Went Wrong”, en la revista online Monday Note, Francia, mayo: http://www.mondaynote.com/2015/05/04/circa-what-went-wrong/ (recuperado el 20/05/2015).

Francis, D., Bessant, J. (2005): "Targeting innovation and implications for capability development”. Technovation 25, Países Bajos, pp. 171-183.

García Avilés, J. A. (2014): “Breve radiografía de la innovación en periodismo”, en el blog MIP UMH, España, mayo: http://mip.umh.es/blog/2014/05/22/radiografia- innovacion/ (recuperado el 20/04/2015).

González Esteban, J. L. (2009): "Modelos de periodismo local y estrategias ante la crisis: el caso del News \& Observer". Revista Latina de Comunicación Social, 64, España, pp. 151-160.

Grupo de Investigación en Comunicación de la Comunidad Valenciana (2015): Ranking de Innovación Periodística 2014. Recuperado el 10 de enero de 2015 de http://www.amic.media/media/files/file 352 723.pdf

Instituto Nacional de Estadística (2014): Encuesta sobre Equipamiento y Uso de Tecnologías de Información y Comunicación en los Hogares. Recuperado el 25 de abril de 2015 de: http://www.ine.es/prensa/np864.pdf

Kamprath, M., Mietzner, D. (2009): “The Nature of Radical Media Innovation - Insights from an Explorative Study", en VVAA, 2nd ISPIM Innovation Symposium - "Stimulating Recovery - The Role of Innovation Management". Nueva York: The Fashion Institute of Technology.

Lindmark, S., Ranaivoson, H., Donders, K., Ballon, P. (2013): “Innovation in Small Regions' Media Sectors. Assessing the Impact of Policy in Flanders", en VVAA, Media Innovations. A Multidisciplinary Study of Change (Coords. T. Storsul y A. Krumsvik). Göteborg: Nordicom, pp. 127-144.

Martínez Molina, M. (2010): "En un escenario complejo de transformación. La crisis de los grandes periódicos". Infoamérica 2, España, pp. 139-148.

OCDE, Eurostat (2006): Guía para la recogida e interpretación de datos sobre innovación. Recuperado el 22 de abril de 2015 de http://www.uis.unesco.org/Library/Documents/OECDOsloManual05 spa.pdf

Pew Research Center (2015): State of the News Media 2015. Recuperado el 15 de febrero de 2015 de http://www.journalism.org/files/2015/04/FINAL-STATE-OF-THE- NEWSMEDIA1.pdf

Schweitzer, T. S. (2003): "Managing interactions between technological and stylistic innovation in the media industries". Technology Analysis \& Strategic Management 15(1), Reino Unido, pp. 1941.

Siapera, E. (2012): Understanding New Media. Londres: SAGE.

Storsul, T., Krumsvik, A. (2013): "What is Media Innovation", en VVAA, Media Innovations. A Multidisciplinary Study of Change (Coords. T. Storsul y A. Krumsvik). Göteborg: Nordicom, pp. 13-26. 
The New York Times (2014). The New York Times Innovation Report. Recuperado el 10 de marzo de 2015 de http://mashable.com/2014/05/16/full-new-york-times- innovation-report/.

Trappel, J. (2015): "What to study when studying media and communication innovation? Research design for the digital age". The Journal of Media Innovations 2(1), Noruega, pp. 7-22 


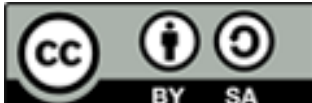

Licencia Creative Commons

Miguel Hernández Communication Journal

mhcj.es

Forma de citar este artículo en las bibliografías

José María Valero Pastor (2015): “Tendencias de la innovación mediática en Estados Unidos” en Miguel Hernández Communication Journal, nº, páginas 161 a 193. Universidad Miguel Hernández, UMH (Elche-Alicante). Recuperado el _ de de 20_ de: [ink del artículo en mhjournal.org] 